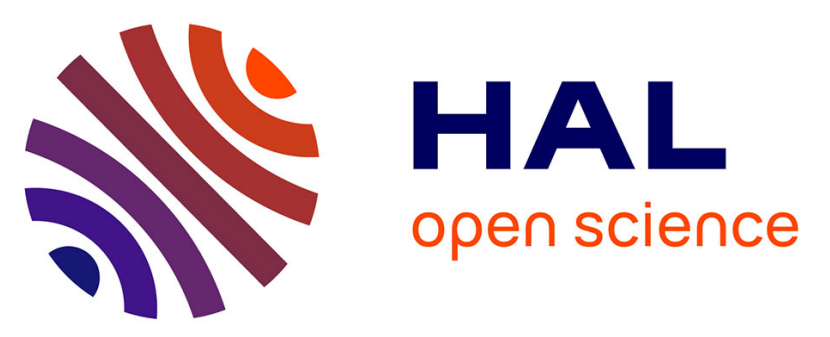

\title{
Long-read assembly of a Great Dane genome highlights the contribution of GC-rich sequence and mobile elements to canine genomes
}

Julia Halo, Amanda L. Pendleton, Feichen Shen, Aurelien J. Doucet, Thomas Derrien, Christophe Hitte, Laura E. Kirby, Bridget Myers, Elzbieta Sliwerska, Sarah Emery, et al.

\section{To cite this version:}

Julia Halo, Amanda L. Pendleton, Feichen Shen, Aurelien J. Doucet, Thomas Derrien, et al.. Longread assembly of a Great Dane genome highlights the contribution of GC-rich sequence and mobile elements to canine genomes. Proceedings of the National Academy of Sciences of the United States of America, 2021, 118 (11), 10.1073/pnas.2016274118 . hal-03206721

\section{HAL Id: hal-03206721 \\ https://hal.science/hal-03206721}

Submitted on 26 May 2021

HAL is a multi-disciplinary open access archive for the deposit and dissemination of scientific research documents, whether they are published or not. The documents may come from teaching and research institutions in France or abroad, or from public or private research centers.
L'archive ouverte pluridisciplinaire $\mathbf{H A L}$, est destinée au dépôt et à la diffusion de documents scientifiques de niveau recherche, publiés ou non, émanant des établissements d'enseignement et de recherche français ou étrangers, des laboratoires publics ou privés.

\section{(2)(1) $\$$}

Distributed under a Creative Commons Attribution - NonCommercial - NoDerivatives $\mid 4.0$ 


\title{
Long-read assembly of a Great Dane genome highlights the contribution of GC-rich sequence and mobile elements to canine genomes
}

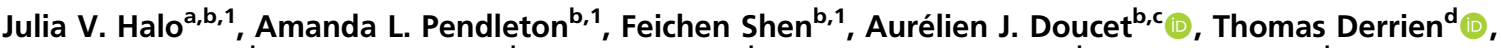

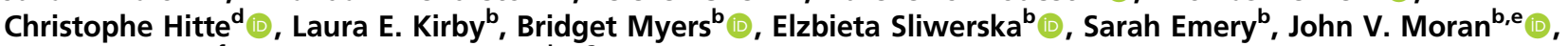 \\ Adam R. Boyko ${ }^{f} \odot$, and Jeffrey M. Kidd ${ }^{b, g, 2}$
}

\begin{abstract}
${ }^{a}$ Department of Biological Sciences, Bowling Green State University, Bowling Green, $\mathrm{OH} 43403$; ${ }^{b}$ Department of Human Genetics, University of Michigan, Ann Arbor, MI 48109; 'Université Côte d'Azur, CNRS, INSERM, Institut de Recherche sur le Cancer et le Vieillissement de Nice, F-06100 Nice, France;

dUniversité de Rennes 1, CNRS, Institut de Génétique et Développement de Rennes-UMR 6290, F-35000 Rennes, France; ${ }^{e}$ Department of Internal Medicine, University of Michigan, Ann Arbor, MI 48109; ' Department of Biomedical Sciences, Cornell University, Ithaca, NY 14850; and ${ }^{9}$ Department Computational Medicine and Bioinformatics, University of Michigan, Ann Arbor, Ml 48109
\end{abstract}

Edited by Mary-Claire King, University of Washington, Seattle, WA, and approved January 25, 2021 (received for review July 31, 2020)

Technological advances have allowed improvements in genome reference sequence assemblies. Here, we combined long- and short-read sequence resources to assemble the genome of a female Great Dane dog. This assembly has improved continuity compared to the existing Boxer-derived (CanFam3.1) reference genome. Annotation of the Great Dane assembly identified 22,182 protein-coding gene models and 7,049 long noncoding RNAs, including 49 protein-coding genes not present in the CanFam3.1 reference. The Great Dane assembly spans the majority of sequence gaps in the CanFam3.1 reference and illustrates that 2,151 gaps overlap the transcription start site of a predicted protein-coding gene. Moreover, a subset of the resolved gaps, which have an $\mathbf{8 0 . 9 5 \%}$ median GC content, localize to transcription start sites and recombination hotspots more often than expected by chance, suggesting the stable canine recombinational landscape has shaped genome architecture. Alignment of the Great Dane and CanFam3.1 assemblies identified 16,834 deletions and 15,621 insertions, as well as 2,665 deletions and 3,493 insertions located on secondary contigs. These structural variants are dominated by retrotransposon insertion/deletion polymorphisms and include 16,221 dimorphic canine short interspersed elements (SINECs) and 1,121 dimorphic long interspersed element-1 sequences (LINE-1_Cfs). Analysis of sequences flanking the $3^{\prime}$ end of LINE-1_Cfs (i.e., LINE-1_Cf 3'-transductions) suggests multiple retrotransposition-competent LINE-1_Cfs segregate among dog populations. Consistent with this conclusion, we demonstrate that a canine LINE-1_Cf element with intact open reading frames can retrotranspose its own RNA and that of a SINEC_Cf consensus sequence in cultured human cells, implicating ongoing retrotransposon activity as a driver of canine genetic variation.

Canis familiaris | long-read assembly | mobile elements | structural variation

T he domestic dog (Canis lupus familiaris) is an established model system for studying the genetic basis of phenotype diversity, assessing the impact of natural and artificial selection on genome architecture, and identifying genes relevant to human disease. The unique genetic structure of dogs, formed as a result of trait selection and breed formation, has particularly aided genetic mapping of dog traits $(1,2)$.

Canine genetics research has taken advantage of a growing collection of genomics tools including high-density singlenucleotide polymorphism arrays, thousands of genome sequences acquired with short-read technologies, the existence of rich phenotype information, and the availability of DNA obtained from ancient samples (3). This research has relied on the reference genome, CanFam, derived from a Boxer breed dog named Tasha and originally released in 2005 (4). The CanFam assembly was constructed at the end of the first phase of mammalian genome sequencing projects and used a whole-genome shotgun approach that included the end-sequencing of large genomic DNA inserts contained within bacterial artificial chromosome (BAC) and fosmid libraries in conjunction with a limited amount of finished BAC clone sequence (4). Subsequent analyses of CanFam and other genomes sequenced in this manner have suggested that there is an incomplete representation of duplicated and repetitive sequences in the resultant assemblies. Although multiple updates have improved the CanFam assembly, yielding the current CanFam3.1 reference assembly (5), numerous assembly errors, sequence gaps, and incomplete

\section{Significance}

Advancements in long-read DNA sequencing technologies provide more comprehensive views of genomes. We used longread sequences to assemble a Great Dane dog genome that provides several improvements over the existing reference derived from a Boxer. Assembly comparisons revealed that gaps in the Boxer assembly often occur at the beginning of protein-coding genes and have a high-GC content, which likely reflects limitations of previous technologies in resolving GCrich sequences. Dimorphic LINE-1 and SINEC retrotransposons represent the predominant differences between the Great Dane and Boxer assemblies. Proof-of-principle experiments demonstrated that expression of a canine LINE-1 could promote the retrotransposition of itself and a SINEC_Cf consensus sequence in cultured human cells. Thus, ongoing retrotransposon activity is a major contributor to canine genetic diversity.

Author contributions: J.V.H., A.L.P., F.S., A.J.D., J.V.M., A.R.B., and J.M.K. designed research; J.V.H., A.L.P., F.S., A.J.D., T.D., C.H., B.M., E.S., S.E., and J.M.K. performed research; J.V.H., A.J.D., B.M., E.S., and S.E. contributed new reagents/analytic tools; J.V.H., A.L.P., F.S., T.D., C.H., L.E.K., and J.M.K. analyzed data; and J.V.H., A.L.P., A.J.D., J.V.M., and J.M.K. wrote the paper.

Competing interest statement: J.V.M. is an inventor on patent US6150160, is a paid consultant for Gilead Sciences, serves on the scientific advisory board of Tessera Therapeutics Inc. (where he is paid as a consultant and has equity options), and currently serves on the American Society of Human Genetics Board of Directors. A.R.B. is the cofounder and Chief Science Officer of Embark Veterinary.

This article is a PNAS Direct Submission.

This open access article is distributed under Creative Commons Attribution-NonCommercialNoDerivatives License 4.0 (CC BY-NC-ND).

1J.V.H., A.L.P., and F.S. contributed equally to this work.

${ }^{2}$ To whom correspondence may be addressed. Email: jmkidd@umich.edu.

This article contains supporting information online at https://www.pnas.org/lookup/suppl/ doi:10.1073/pnas.2016274118/-/DCSupplemental.

Published March 8, 2021. 
gene models remain. Thus, a more complete and comprehensive dog genome sequence will aid the identification of mutations that cause phenotypic differences among dogs and enable continued advances in comparative genomics (6).

Genome analyses have revealed that canine genomes contain an elevated number of high GC content segments relative to other mammalian species $(7,8)$. Genetic recombination may contribute to the evolution of these segments. Studies across a number of mammalian species have indicated that genetic recombination events cluster in specific regions known as hotspots (9). In many species, the PRDM9 zinc finger protein binds to specific nucleotide sequences to promote the initiation of recombination (10-12). In addition to cross-overs, the molecular resolution of recombination events involves gene conversion (13). Gene conversion shows a bias in favor of copying G/C sequences and makes an important contribution to the evolution of genome content $(14,15)$. Due to gene conversion events and changes in the DNA binding domains of PRDM9, the locations of recombination hotspots in many species are not stable over evolutionary time $(16,17)$. However, dogs lack a functional PRDM9 protein (18), and canine recombination maps indicate that recombination events are concentrated in GC-rich segments that reside near gene promotors $(19,20)$. Thus, the observed distribution of GC-rich sequence segments in the canine genome may be a consequence of the stable recombination landscape in canines.

Analysis of the CanFam3.1 reference has demonstrated that a large fraction of the dog genome has resulted from the expansion of transposable elements belonging to the short and long interspersed element (SINE and LINE) families. Fine mapping has implicated mobile element insertions, and associated events such as retrogene insertions, as the causal mutation underlying morphological differences, canine diseases, and selectively bred phenotypes (21-32). A comparison between the Boxer-derived CanFam reference and a low coverage $(\sim 1.5 \times)$ draft genome from a Poodle identified several thousand dimorphic copies of a recently active lysine transfer RNA-derived canine SINE (SINEC) element, SINEC_Cf, implying a variation rate 10- to 100 -fold higher than observed for still-active SINE lineages in humans (33). Similarly, insertions derived from a young canine LINE-1 lineage, L1_Cf, were found to be greater than threefold more prevalent than L1Hs, the active LINE-1 lineage found in humans $(33,34)$. However, the assembly of long repetitive sequences with a high nucleotide identity is technically challenging, leaving many LINE sequences incorrectly represented in existing reference genomes. Consequently, the biological impact of these elements has remained largely unexplored, and the discovery of dimorphic canine LINE-1 sequences is limited to a few reports $(31,33,35,36)$.

Following the era of capillary sequencing, genome reference construction shifted toward high coverage assemblies that utilized comparatively short sequencing reads. These approaches offered a great reduction in cost and an increase in per-base accuracy, but still were largely unable to resolve duplicated and repetitive sequences, often yielding assemblies that contained tens of thousands of contigs (37). Methods based on linked-read or chromosome conformation sequencing are capable of linking the resulting contigs into larger scaffolds, including entire chromosome arms, but these scaffolds are typically littered with sequence gaps reflecting the poor representation of repetitive sequences (38-40). Here, we analyze the genome of a female Great Dane named Zoey that we sequenced using PacBio longread technology. We integrated this long-read data with additional sequencing resources, including standard high-coverage short-read sequence data, as well as sequence data derived from mate-pair and pooled fosmid libraries, to generate a highquality assembly. Using this new assembly, we annotate gene structures and GC-rich sequences that are absent from
CanFam3.1 and underrepresented in existing Illumina canine short-read sequence datasets. We demonstrate that gaps in the CanFam3.1 assembly are enriched with sequences that have an extremely high GC content and that overlap with transcription start sites and recombination hotspots. We identify thousands of mobile element insertions, including intact LINE-1 copies, and make use of our fosmid library to subclone an intact L1 Cf element. We demonstrate that a cloned canine L1_Cf is capable of high levels of retrotransposition of its own mRNA (in cis) and can drive the retrotransposition of a consensus SINEC_Cf RNA (in trans) in cultured human cells. Our analysis provides a more complete view of the canine genome and demonstrates that the distribution of extremely GC-rich sequences and the activity of mobile elements are major factors affecting the content of canine genomes.

\section{Results}

Long-Read Assembly of a Great Dane Genome. We performed a genome assembly of a female Great Dane, Zoey, using multiple genome sequencing resources that included a standard Illumina short-read sequencing library, a 3-kb Illumina mate-pair sequencing library, sequences from a pooled fosmid library, and $\sim 50 \times$ raw long-read coverage generated using the PacBio RSII system. PacBio long reads were assembled using the Falcon assembler (41), yielding 2,620 primary contigs longer than $1 \mathrm{kbp}$ that encompassed $2.3 \mathrm{Gbp}$ of sequence. In addition, $6,857 \mathrm{sec}$ ondary contigs, with a total length of $178.5 \mathrm{Mbp}$, that represent the sequence of heterozygous alleles were assembled ( $S I \mathrm{Ap}$ pendix, section 1).

The assembly process is based on detecting overlaps among sequencing reads. As a result, reads that end in long stretches of sequence which map to multiple genomic locations and that have high sequence identity can give rise to chimeric contigs that falsely conjoin discontinuous genomic segments. Using Illumina mate-pair and fosmid pool data from Zoey, clone end sequences from the Boxer Tasha, and alignments to the existing CanFam3.1 assembly, we identified 20 contigs that appeared to be chimeric. We split these contigs at the chimeric junctions, yielding a total of 2,640 contigs with the shortest contig length required to cover $50 \%$ of the genome $\left(\mathrm{N}_{50}\right)$ of $4.3 \mathrm{Mbp}$ and a maximum contig length of $28.8 \mathrm{Mbp}$. As expected, alignment against the CanFam3.1 assembly indicated comprehensive chromosome coverage. Consistent with the problems in assembly caused by segmental duplications, we found that long contigs (>3 Mbp) ended in duplicated sequence greater than $10 \mathrm{kbp}$ more often than expected by chance $(P<0.001$ by permutation; see $S I A p$ pendix, section 1).

Alignment of the 2,640 contigs and the raw PacBio reads against the CanFam3.1 assembly revealed apparent gaps between contigs, many of which were spanned by PacBio reads. Reasoning that these reads may have been excluded from the assembly due to length cutoff parameters used in the Falcon pipeline, we performed a locus-specific assembly utilizing the Canu assembler (v1.3) (42). This process yielded 373 additional contigs with a total length of $10.5 \mathrm{Mbp}$ and an $\mathrm{N}_{50}$ length of 30 kbp. Based on the mapping of the Zoey derived mate-pair sequences and end sequences from the Tasha-derived fosmid and BAC libraries, we linked the 2,640 primary contigs and 373 gapfilling contigs into scaffolds (43). Gap-filling contigs that were not linked using paired reads were excluded from further analysis, resulting in a total of 1,759 scaffolds with an $\mathrm{N}_{50}$ of $21 \mathrm{Mbp}$. Scaffolds were assigned to chromosomes and ordered based on alignment to CanFam3.1 (Fig. 1). Sequences that appeared to represent allelic variants based on sequence identity and read depth were removed, yielding a chromosomal representation that included 754 unlocalized sequences (SI Appendix, section 1). 

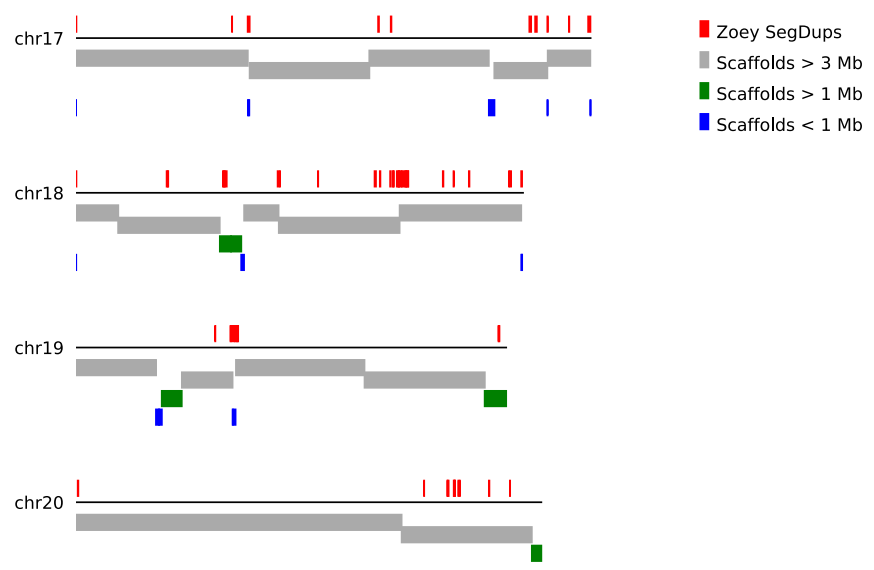

$\begin{array}{llrrrr}20 & 30 & 40 & 50 & 60 & 70\end{array}$

Fig. 1. Alignment of assembled scaffolds to the CanFam3.1 genome. Each of the assembled scaffolds was aligned to the CanFam3.1 reference genome. Results are shown for four chromosomes. The colored bars below each line indicate the corresponding position of each scaffold, colored based on their indicated length. Above each line, regions of segmental duplications based on read depth in the Zoey Illumina data are indicated by red boxes.

Annotation of Genome Features. We identified segmental duplications in the Zoey and CanFam3.1 assemblies based on assembly self-alignment (44) and read depth (45) approaches (SI Appendix, section 3). Although the number of duplications is similar in each genome, the Zoey assembly contains a smaller total amount of sequence classified as "duplicated," which likely reflects the continued challenges in properly resolving duplications longer than $10 \mathrm{kbp}$ (Table 1) (46). To compare the largescale organization of the assemblies, we constructed reciprocal liftOver tracks that identify corresponding segments between the CanFam3.1 and Zoey assemblies (47). Based on this comparison, we identified 44 candidate inversions of $>5 \mathrm{~kb}$. Of these candidate large inversions, $68 \%$ (30 of 44) were associated with duplicated sequences. The X chromosome, which contributes $5.3 \%$ of the genome length, contains $41 \%(18 / 44)$ of the predicted inversions.

We created a gene annotation based on previously published RNA sequencing data using both genome-guided and genomefree approaches (48-50) (SI Appendix, section 2). Following filtration, this process resulted in a final set of 22,182 proteincoding gene models; 49 of these gene models are absent from the CanFam3.1 assembly. Full-length matches were found for only $84.9 \%(18,834)$ of all protein-coding gene models, while near-full-length alignments were found for $93 \%(20,670)$ of the models. We additionally annotated 7,049 long noncoding RNAs (51), including 84 with no or only partial alignment to CanFam3.1. Using existing RNA sequencing (RNA-Seq) data (5), we estimated expression values for each protein-coding gene across 11 tissues and report the results as tracks on a custom University of California, Santa Cruz (UCSC) Genome Browser assembly hub (52) (Fig. 2). The assembly hub illustrates correspondence between the CanFam3.1 and Zoey assemblies and displays the annotation of additional features including structural variants, segmental duplications, common repeats, and BAC clone end sequences (SI Appendix, section 7).

Resolved Assembly Gaps Include GC-Rich Segments Underrepresented in Illumina Libraries. Alignment indicates that 12,806 of the autosomal gaps in CanFam3.1 are confidently localized to a unique location in the Zoey genome assembly. In total, $16.8 \%(2,151)$ of the gap segments overlap with a transcription start site of a protein-coding gene, which makes it possible to better understand the importance of these previously missing sequences in canine biology $(5,53)$. Surprisingly, analysis of unique k-mer sequences that map to the CanFam3.1 gap sequences suggested that these DNA segments often are absent from existing Illumina short-read datasets, even though analysis of DNA from the same samples using a custom array comparative genomic hybridization platform indicates their presence. Interrogation of read-pair signatures also suggests that these sequences are systematically depleted in Illumina libraries, which is due to their extreme GC-rich sequence composition (SI Appendix, section 4).

The sequences corresponding to gaps in CanFam3.1 have an extremely high GC content, with a median GC content of $67.3 \%$, a value substantially higher than the genome-wide expectation of $39.6 \%$ (Fig. 3). Given the relationship between GC content and recombination in dogs $(19,20)$, we examined the distance between CanFam3.1 gap sequences and recombination hotspots. We found that $11.8 \%$ of gap segments $(1,457$ of 12,304 on the autosomes) are located within $1 \mathrm{kbp}$ of a hotspot, compared to only $2.9 \%$ of intervals expected by chance. These patterns are driven by a subset of segments that have the most extreme GC content. We identified 5,553 segments with a GC content greater than that obtained from 1,000 random permutations. These extreme GC segments span a total of $4.03 \mathrm{Mbp}$ in the Zoey assembly, have a median length of $531 \mathrm{bp}$, a median GC content of $80.95 \%$, and are located much closer to transcription start sites (median distance of $290 \mathrm{bp}$ ) and recombination hotspots (median distance of $68.7 \mathrm{kbp}$ ) than expected by chance.

Table 1. Comparison of the Boxer and Great Dane assemblies

\begin{tabular}{lrr} 
& CanFam3.1 autosomes $+X$ & Zoey autosomes $+X$ \\
\hline Total length & $2,327,633,984$ & $2,326,329,672$ \\
$\quad$ Non- $N$ & $2,317,593,971$ & $2,320,292,846$ \\
Number of gaps & 19,553 & 997 \\
Longest contiguous segment & $2,428,071$ & $28,813,894$ \\
Mean contiguous segment length & 118,523 & $2,239,665$ \\
Median contiguous segment length & 54,641 & $2,107,836$ \\
$N_{50}$ segment length & 277,468 & $4,765,928$ \\
Segmental duplications genome alignment, $>1$ kbp, $>90 \%$ ID & 6,371 \\
$\quad$ bp & $49,339,683$ & 450 \\
Segmental duplications Penelope read depth & 459 & 425,166 \\
$\quad$ bp & $47,757,534$ & $40,836,807$ \\
\hline
\end{tabular}

Presented are general assembly statistics for the primary autosomal and $\mathrm{X}$ chromosome sequence of the CanFam3.1 and Zoey assemblies. Contiguous segment refers to the length of sequence uninterrupted by an " $\mathrm{N}$ " nucleotide. Segmental duplications were identified in each assembly based on an assembly self-alignment and by the depth of coverage of Illumina sequencing reads from Penelope, an Iberian Wolf. See SI Appendix, section 3 for additional details. 


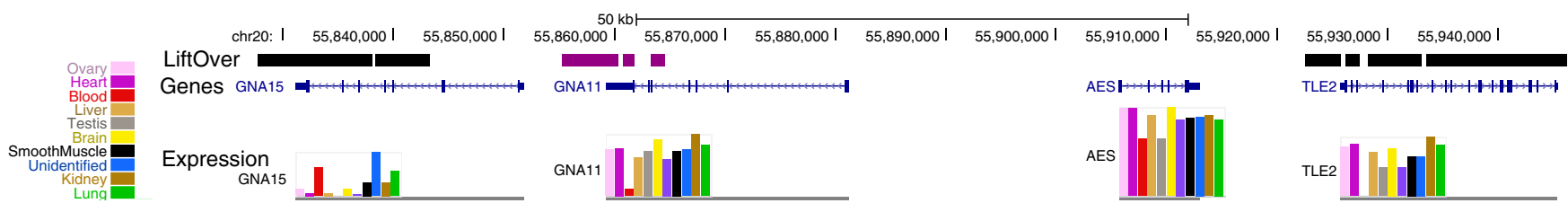

Fig. 2. Annotation of genes missing from the CanFam3.1 assembly. A genome browser view of chr20 on the Zoey assembly is shown. The top track summarizes a comparison between the Zoey and CanFam3.1 assemblies using the UCSC liftOver tool. Black segments show alignment to the corresponding chromosome on the CanFam3.1 assembly. Purple segments match to an unlocalized contig (chrUn_JH374124) in the CanFam3.1 assembly. The large region in the middle between the purple and black segments is absent from the CanFam3.1 assembly. The track below shows the position of four genes in this region annotated using RNA-Seq data: GNA15, GNA11, AES, and TLE2. The colored bars below each gene model show the expression levels across different tissues, as indicated by the color key at the left. See SI Appendix, section 2 for additional details.

Mobile Elements Account for the Majority of Structural Differences between Canine Genomes. We compared the CanFam3.1 and Zoey assemblies to identify insertion-deletion differences at least $50 \mathrm{bp}$ in length. After filtering variants that intersect with assembly gaps or segmental duplications, we identified 16,834 deletions (median size: $207 \mathrm{bp}$ ) and 15,621 insertions (median size: 204 bp) in the Zoey assembly relative to CanFam3.1 (SI Appendix, section 5). In total, these structural variants represent 13.2 Mbp of sequence difference between the two assemblies. The length distribution of the detected variants shows a striking bimodal pattern, with clear peaks at $\sim 200 \mathrm{bp}$ and $\sim 6 \mathrm{kbp}$, consistent with the size of SINEC and LINE-1 sequences (Fig. 4). We inspected the sequence of the events in the 150- to 250-bp size range and found that 7,298 deletions and 6,071 insertions were dimorphic SINEC sequences. Additionally, LINE-1 sequences accounted for 339 deletions and 581 insertions longer than $1 \mathrm{kbp}$.

Our assembly also contains 6,857 secondary contigs, which represent alternative sequences at loci where Zoey is heterozygous for a structural variant. Alignment of these secondary contigs against the CanFam3.1 assembly yielded an additional

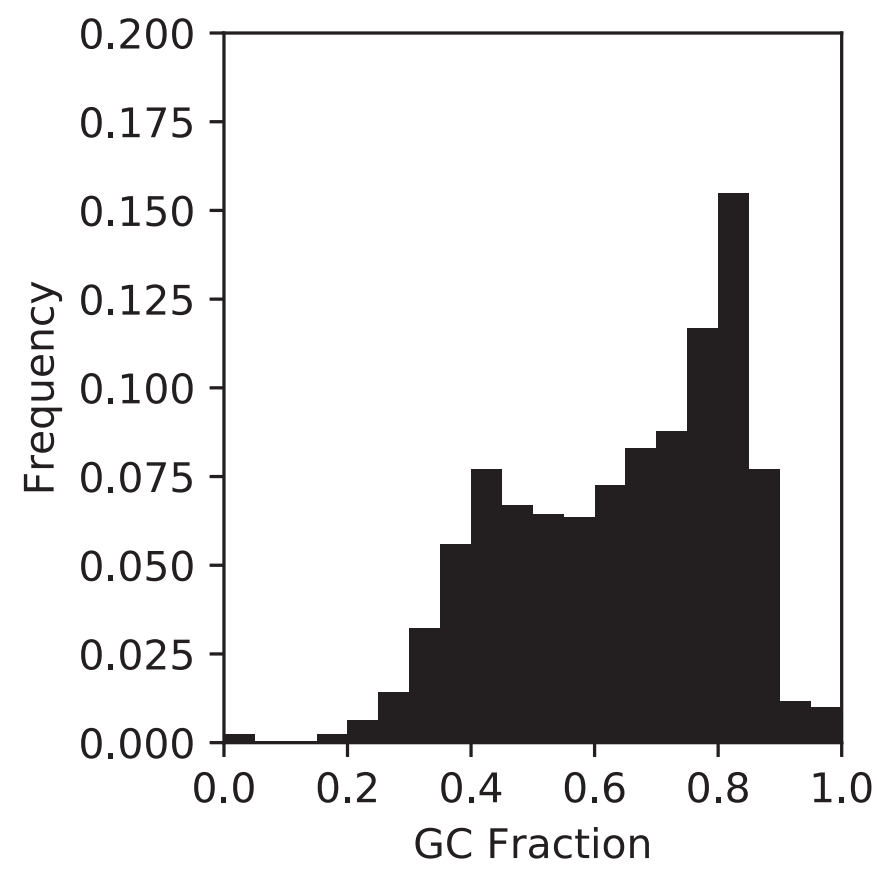

Fig. 3. CanFam3.1 assembly gaps are enriched for sequence with extreme GC content. Depicted is the distribution of GC content for 12,806 resolved assembly gaps. A subset consisting of 5,553 of the 12,806 segments have a GC content greater than that found in $99 \%$ of randomly selected segments. See SI Appendix, section 4 for additional details.
2,665 deletion and 3,493 insertion events, encompassing a total of $2.67 \mathrm{Mbp}$ of sequence. We further inspected the sequence of these variants and found 1,259 deletions and 1,593 insertions consistent with dimorphic SINEC elements, and 75 deletions and 126 insertions consistent with dimorphic LINE-1 elements. Together, comparison of the Zoey and CanFam3.1 genomes identified at least 16,221 dimorphic SINEC and 1,121 dimorphic LINE-1 sequences (SI Appendix, section 5). We assessed these variants for the hallmarks of retrotransposition. The reported dimorphic SINECs and LINE-1s are flanked by target site duplications that have a median size of $15 \mathrm{bp}$ and end in poly(A) tracts with a median length of 9 bp to $12 \mathrm{bp}$. The LINE-1s contain the longest poly(A) tracts and also show more variation in tract length. This difference in poly(A) lengths reflects differences between LINE-1 and SINEC transcripts: LINE-1 transcripts are polyadenylated (54), whereas SINE poly(A) tracts are encoded by the source element (55). We additionally analyzed the inferred endonuclease recognition site and found the expected consensus of 5'-TTTTT/AA (56-58) (SI Appendix, section 5).

Gene conversion plays an important role in the evolution of SINE sequences and can result in the apparent replacement of an older, diverged SINE with sequence derived from a less diverged element (59-62). To confirm the origin of the detected insertions, we searched the empty-site sequence of each dimorphic SINEC locus against the genome of the Dhole (Cuon alpinus, accession GWHAAAC00000000) (63), a species estimated to have shared a common ancestor with dogs $\sim 3$ million to 7 million years ago $(64,65)$. We found that $94 \%$ of query sequences have a contiguous match against the Dhole genome, indicating the vast majority of dimorphic SINECs inserted in the dog lineage following the split from the Dhole common ancestor (SI Appendix, section 5).

LINE-1 transcription often bypasses the polyadenylation signal encoded within the element, resulting in the inclusion of flanking genomic sequence in the LINE-1 RNA (66-69). Thus, after retrotransposition, the resulting 3 '-transductions can be used as sequence signatures to identify the progenitor source elements of individual LINE-1 insertions $(70,71)$. We identified 18 transduced sequences among the dimorphic LINE-1 sequences in our dataset. Of these transduced sequences, 17 aligned elsewhere in the genome at a location that is not adjacent to an annotated LINE-1. This includes a pair of LINE-1 copies on chr25 and chrX which share the same transduced sequence, as well as a locus on chr19 that has the same transduction as a duplicated sequence present on chr2 and chr3. Such "parentless" 3' transductions suggest the presence of additional dimorphic LINE-1 sequences that are capable of retrotransposition (SI Appendix, section 5).

Canine Genomes Contain LINE-1s and SINEs Capable of Retrotransposition. The high degree of dimorphic LINE-1 and SINEC sequences found between the two assemblies suggests 
A

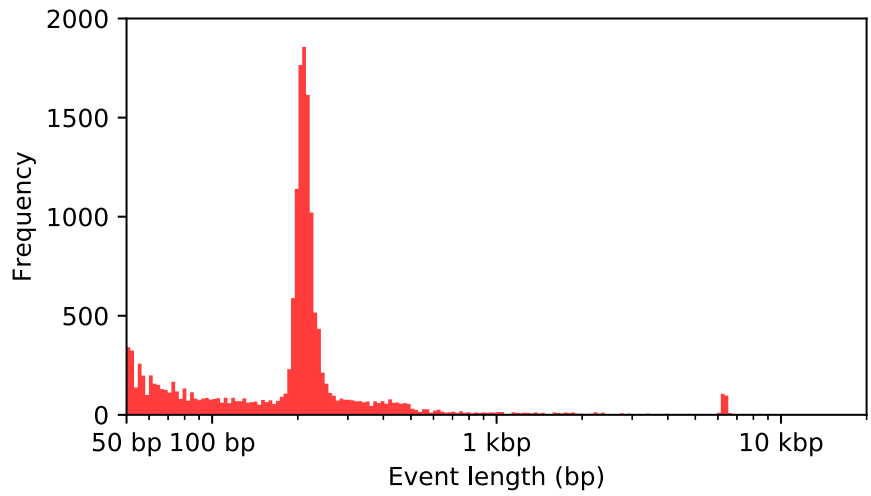

B

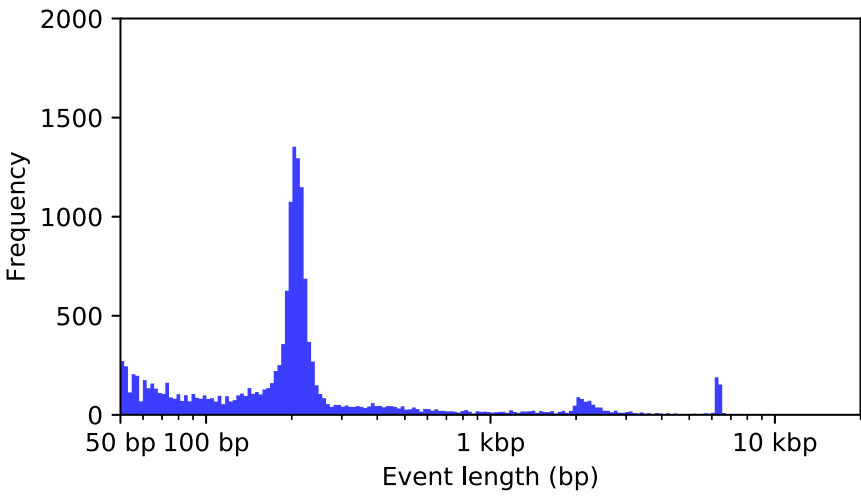

Fig. 4. Size of structural variants identified between the CanFam3.1 and Zoey assemblies. Shown are histograms depicting the size distribution of $(A) 16,834$ deletions and $(B)$ 15,621 insertions between the Zoey and CanFam3.1 assemblies. Variant size is plotted on a logarithmic scale such that the bins in the histogram are of equal size in the log scale. Large increases at $\sim 200$ bp and $\sim 6 \mathrm{kbp}$ indicate the disproportionate contribution of dimorphic LINE1 and SINEC sequences to the genetic differences between the two assemblies. See SI Appendix, section 5 for additional details.

that mobile element activity represents a mutational process that is ongoing in canines. The canine LINE-1 (L1_Cf) consensus sequence contains segments of GC-rich sequence and homopolymer runs, including a stretch of 7 " $\mathrm{C}$ " nucleotides in the ORF1p coding sequence that likely are prone to errors incurred during DNA replication, PCR, and sequencing. Thus, a bioinformatic search for L1_Cf sequences with intact open reading frames is biased by uncorrected sequencing errors. We therefore searched the Zoey assembly for sequences that have long matches with low sequence divergence from the L1 Cf consensus. The Zoey assembly encodes 837 L1_Cf sequences that have less than $2 \%$ divergence and are greater than $99.4 \%$ of full length; an additional 169 elements are present on the secondary contigs. This set includes 187 full-length LINE-1s, of which 31 were found in secondary contigs. For comparison, these values represent a $65 \%$ increase over the 113 elements present in CanFam3.1 that meet the same criteria (SI Appendix, section 5).

To more thoroughly characterize canine LINE-1 copies that may remain active, we isolated and sequenced individual fosmids from Zoey predicted to contain full-length LINE-1s. We identified one sequence, from fosmid clone 104_5 on chr1 (L1_Cf104_5), possessing intact open reading frames, which encode the $\mathrm{OR} \overline{\mathrm{F}} 1 \mathrm{p}$ and $\mathrm{ORF} 2 \mathrm{p}$ predicted proteins, that lack mutations expected to disrupt protein function (SI Appendix, section 6). We subcloned this element for functional analysis in a cultured cell assay that uses an indicator cassette that is only expressed following a successful round of retrotransposition (72-74), yielding G418-resistant foci. We found that the L1_Cf-104_5 element is capable of retrotransposition of its own m $\bar{R} N A$ in $c i s$ in human HeLa cells (Fig. 5 and SI Appendix, section 6).

SINE sequences are nonautonomous elements that utilize the function of LINE-1 ORF2p to mediate their retrotransposition in trans $(74,75)$. To test the capability of L1_Cf-104_5 to mobilize SINE RNA in trans, we constructed a second reporter vector containing the SINEC_Cf consensus sequence marked by an appropriate indicator cassette (75). We found that expression of L1_Cf-104_5 was capable of mobilizing both canine SINEC and human Alu RNAs in trans (Fig. 5 and SI Appendix, section 6).

\section{Discussion}

Due to their unique breed structure, history of selection for disparate traits, and extensive phenotypic data, dogs are an essential model for dissecting the genetic basis of complex traits and understanding the impact of evolutionary forces on genome diversity. The era of long-read sequencing is revolutionizing genomics by enabling a more complete view of genomic variation (76). Here, we describe the assembly and annotation of the genome of a Great Dane dog and compare it with the Boxerderived CanFam3.1 reference assembly. Comparison of our Great Dane genome to the CanFam3.1 reference revealed several key findings important to canine genome biology. Several other long-read assemblies of canines are planned or have been recently released $(77,78)$. The availability of these resources will provide significant benefits to the canine genomics community.

Our Great Dane assembly has improved sequence continuity, resolves novel gene structures, and identifies several features important to canine genome biology. For example, we created a gene annotation that includes 49 predicted protein-coding genes that are absent from the CanFam3.1 reference genome. Our analysis also identified 2,151 protein-coding gene models whose transcription start position corresponds to a gap in the CanFam3.1 assembly. This finding largely resolves prior observations that many dog genes appear to have incomplete first exons and promoters $(5,6)$. Analysis of the Great Dane assembly further revealed that gaps in the CanFam3.1 assembly are enriched for sequence that has extremely high-GC content, providing a probable explanation for their absence from the CanFam3.1 assembly (79).

The presence of extremely GC-rich segments likely reflects a key aspect of canine genome biology. In contrast to humans and many other mammals, genetic recombination in canines is targeted toward gene promoter regions, due to the absence of a functional PRDM9 gene (20). In other species, the PRDM9 protein binds to specific nucleotide sequences and targets the initiation of recombination to distinct loci in the genome. It has been hypothesized that recombination in dogs is instead localized by general chromatin marks, which are associated with promoters, resulting in a fine-scale genetic map that is more stable over evolutionary time $(19,20)$. In addition to crossing over, recombination events result in gene conversion, a process with a bias in favor of $\mathrm{G} / \mathrm{C}$ alleles. Biased gene conversion can be modeled as positive selection in favor of $\mathrm{G} / \mathrm{C}$ alleles at a locus $(14,15,80)$ and has been previously proposed as an explanation for the unusual GC content of the dog genome $(19,20)$. Our analysis indicates that the GC-rich segments associated with recombination hotspots are larger than expected previously. These expanded segments have an unknown effect on the expression of their associated gene, have been largely absent from previous genome assemblies, and are depleted from Illumina sequencing 
A

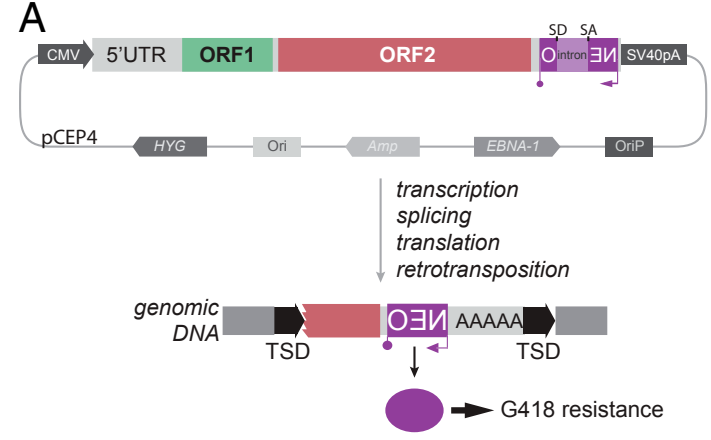

JM101/L1.3

(L1Hs)

JM105/L1.3

(L1Hs, RT-)

L1Cf-104_5

(L1Cf)
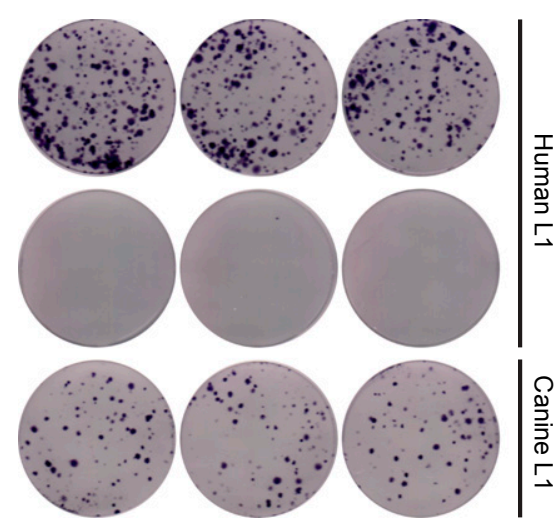

\section{工}

B

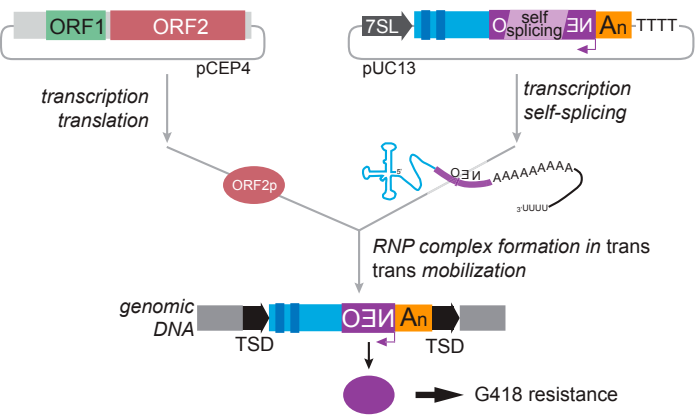

JM101/L1.3 Aneo (L1Hs) .

JM105/L1.3 $\Delta$ neo (L1Hs, RT-)

L1Cf-104 $5 \Delta$ neo (L1Cf)

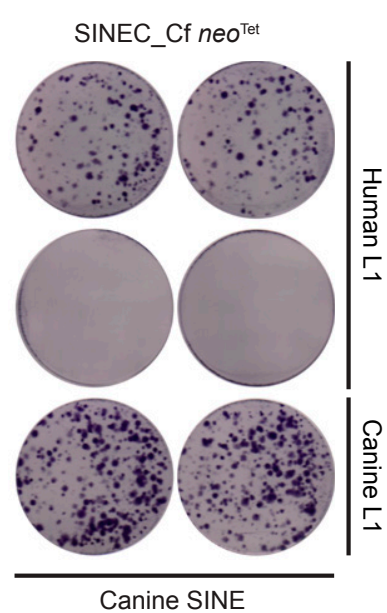

Fig. 5. Identification of canine LINE-1 and SINEC elements capable of retrotransposition. (A) Top) A full-length L1_Cf equipped with a retrotransposition indicator cassette $(\mathrm{mneol})$ was assayed for retrotransposition in human HeLa-HA cells. TSD indicates a target site duplication generated upon retrotransposition. (Bottom) Results of the retrotransposition assay. JM101/L1.3 (positive control) contains an active human LINE-1. JM105/L1.3 (negative control) contains a human LINE-1 that harbors an inactivating missense mutation in the reverse transcriptase domain of ORF2p (99). ADL1Cf-104_5 contains the fulllength canine LINE-1 identified in this study. (B) (Top) A consensus SINEC_Cf element equipped with an indicator cassette to monitor the retrotransposition of RNA pol III transcripts (neo ${ }^{\text {tet }}$ ) (75) was assayed for retrotransposition in human HeLa-HA cells in the presence of either an active human LINE-1 or the newly cloned L1_Cf-104_5 sequence that lacks a retrotransposition indicator cassette (JM101/L1.3Aneo or ADL1Cf-104_5 $\Delta$ neo, respectively). (Bottom) Results of the retrotransposition assay. JM101/L1.3 3 neo (positive control) contains an active human LINE-1. JM105/L1.3 $\Delta$ neo (negative control) contains a human LINE-1 that harbors an inactivating missense mutation in the reverse transcriptase domain of ORF2p (99). ADL1Cf-104_5 $\Delta$ neo contains an active canine LINE-1 (see A). The expression of either JM101/L1.3 $\Delta$ neo or ADL1-Cf-105 $\Delta$ neo could drive human Alu and canine SINEC_Cf retrotransposition. In both assays, the bluestained foci represent G418-resistant foci containing a presumptive retrotransposition event. See SI Appendix, section 6 for additional details.

data. A more extensive examination of the long-term consequence of stable recombination hotspots on genome sequence structure will require assessment of genomes of other species which lack PRDM9 using long-read technologies.

Long-read sequencing offers a less biased view of structural variation between genomes, particularly for insertions (81). The profile of genomic structural variation between the Zoey and CanFam3.1 assemblies is dominated by dimorphic SINEC and LINE-1 sequences, with 16,221 dimorphic SINEC and 1,121 dimorphic LINE-1 sequences. Although analogies between humans and dogs can be problematic (82), a comparison with humans illustrates the magnitude of the mobile element diversity found between the Great Dane and Boxer genome assemblies. In terms of human mobile element diversity, the 1000 Genomes Project estimates that an individual differs from the reference genome by an average of 915 Alu insertions and 128 LINE-1 insertions (83). A recent study collated these findings, along with other published datasets, and identified a total of 13,572 dimorphic Alu elements in humans (84), although we note that these estimates are based on Illumina sequencing data, which have limitations in mapping to repetitive regions and in fully capturing insertion alleles (81). Finally, an approach specifically designed to identify dimorphic human LINE-1 insertions utilizing long-read sequencing data identified 203 nonreference insertions in the benchmark sample NA12878, of which 123 which were greater than $1 \mathrm{kbp}$ in length (85).

Illumina sequencing data indicate that Zoey differs from the CanFam3.1 reference at 3.57 million single-nucleotide variants (SNVs). This number is lower than the number of differences typically found in a globally diverse collection of human genomes (4.1 million to 5.0 million SNVs) (83), and is comparable to the number found in the National Heart, Lung, and Blood Institute's (NHLBI) TOPMed dataset (86) (median of 3.3 million SNVs among 53,831 humans sequenced as part of the NHLBI's TransOmics for Precision Medicine program). Relative to the number of SNVs, the level of LINE-1 and SINEC dimorphism we found between two dog genomes is disproportionately large. This total represents an $\sim 17$-fold increase in SINE differences (16,221/915) and an eightfold increase in LINE differences $(1,121 / 128)$ compared to the numbers found among humans. Remarkably, more dimorphic SINEs were found between these two breed dogs than have been found in studies of thousands of humans $(84,87)$. Our data will aid systematic studies of the potential contribution of these elements to canine phenotypes, including cancers (88).

Comparison with the Dhole genome suggests that most of the dimorphic SINEC insertions occurred in the last few million 
years. Given the high copy number of SINECs in canine genomes, and the unique features of genetic recombination in canines, gene conversion may play an important role in the evolution of SINEC sequence (59-62). The high activity level of SINEC further complicates evolutionary comparisons. For example, we identified independent insertions of distinct SINECs at nearly the same position in a dog and a Dhole genome. Such cooccurrences complicate the genotyping of SINEC insertions across species and should be accounted for in comparative analyses.

Further study is required to determine the relative contribution of 1) new insertions in breeds or populations versus 2) the assortment of segregating variants that were present in the progenitor populations. However, our study suggests that retrotransposition is an ongoing process that continues to affect the canine genome. We provide proof-of-principle evidence that dog genomes contain LINE-1 and SINEC elements that are capable of retrotransposition in a cultured cell assay. We also identified two LINE-1 lineages with the same $3^{\prime}$ transduced sequence associated with multiple elements, suggesting the presence of multiple canine LINE-1s that are capable of spawning new insertions. Additionally, analysis of $3^{\prime}$ transduction patterns suggests the presence of additional active LINE-1s in canines that have yet to be characterized. Thus, a full understanding of canine evolution and phenotypic differences requires consideration of these important drivers of genome diversity.

\section{Methods}

Genome assembly and analysis utilized long-read and short data from a female Great Dane named Zoey, a pooled fosmid library (89) constructed from Zoey, sequence data generated from a female Boxer, named Tasha, as part of the CanFam genome assembly (4), and results from a custom comparative genomics hybridization array (array-CGH). Data accessions and detailed methods are available in SI Appendix.

Genome assembly of $\sim 50$-fold whole-genome, single-molecule, real-time sequencing data from Zoey was performed on DNAnexus using the Falcon 1.7.7 pipeline (41) and the Damasker suite (90). Chimeric contigs were identified based on mapped reads from the Zoey mate-pair jumping library, the Zoey fosmid pools, the Tasha BAC end sequences, and Tasha fosmid end sequences. Regions that showed a lack of concordant paired end coverage were identified as potential chimeric junction sites and split apart prior to scaffolding. Primary contigs were supplemented with contigs obtained from a local assembly of reads aligning to gaps between contigs on CanFam3. using Canu v1.3 (42). Contigs were linked into scaffolds using mapping of the Zoey mate data, Tasha BAC end sequence data, and Tasha fosmid data using the BESST scaffolding algorithm (version 2.2.7) (43) and assigned to chromosomes based on alignment to CanFam3.1. Chain files for use with the UCSC liftOver tool were constructed based on blat (47) alignments. A UCSC TrackHub hosting the Zoey assembly, as well as relevant annotations of both the Zoey assembly and CanFam3.1, is available at https://github.com/ KiddLab/zoey_genome_hub.

Common repeats in both the CanFam3.1 and Zoey assemblies were identified using RepeatMasker version 4.0.7 with option "-species dog," using the rmblastn (version 2.2.27+) search engine and a combined repeat database consisting of the Dfam_Consensus-20170127 and RepBase20170127 releases. Self-alignment analysis of each assembly was performed using SEDEF (44) with default parameters. Results were filtered for alignments at least $1 \mathrm{~kb}$ in length and at least $90 \%$ sequence identity. Read depth analysis was performed using fastCN as described previously (45). Copy number estimates were constructed in nonoverlapping windows each containing $3 \mathrm{kbp}$ of unmasked sequence. Segmental duplications were identified as runs of four windows in a row with an estimated copy number

1. E. K. Karlsson, K. Lindblad-Toh, Leader of the pack: Gene mapping in dogs and other model organisms. Nat. Rev. Genet. 9, 713-725 (2008).

2. A. R. Boyko, The domestic dog: Man's best friend in the genomic era. Genome Biol. 12, 216 (2011).

3. E. A. Ostrander, R. K. Wayne, A. H. Freedman, B. W. Davis, Demographic history, selection and functional diversity of the canine genome. Nat. Rev. Genet. 18, 705-720 (2017).

4. K. Lindblad-Toh et al., Genome sequence, comparative analysis and haplotype structure of the domestic dog. Nature 438, 803-819 (2005). of $\geq 2.5$. To provide an unbiased assessment of duplication content, read depth analysis was performed based on Illumina data form Penelope, an Iberian Wolf, in addition to sequences from Zoey and Tasha.

Forty-two canine RNA-Seq runs representing 11 tissue types were used to annotate genes in the Zoey genome (5). De novo gene models were created based on alignment of RNA-Seq reads using Cufflinks $(v 2.2 .1)(48,49)$ and, in a non-reference-guided fashion, using Trinity (v2.3.2) (50). Gene models were merged and annotated using PASA-Lite (91) and the transdecoder pipeline (version 5.0.1) (92). Gene names and functional annotations were determined using BLAST2GO (93). Expression levels for each of the 22,182 protein-coding gene models were estimated using Kallisto (version 0.46.0) (94). Long noncoding RNAs in the Zoey genome were identified using the FEELnc program (51).

To identify large insertion and deletion variants, the Zoey assembly and 6,857 secondary contigs were aligned to CanFam3.1 using minimap2 (version 2.9-r720) with the -asm5 option (95). The output from the alignment was parsed using the paftools.js program released as part of minimap2 to identify candidate variants. Breakpoint coordinates were refined by performing targeted alignment of the flanking and variant sequence for each candidate using AGE (96).

Individual fosmids containing potentially full-length L1_Cf elements were isolated from pools using a lifting procedure coupled with hybridization of a probe containing digoxygenin-labeled dUTP. Isolated fosmids were sequenced in small pools via RS II PacBio sequencing and assembled using the HGAP2 software (97). An intact L1_Cf was subcloned from fosmid 104_5, equipped with an $m n e o l$ retrotransposition indicator cassette, and tested for retrotransposition in HeLa-HA cells $(72,73)$. The construction of the L1_Cf expression vector and the conditions used to assay for retrotransposition are detailed in SI Appendix, section 6.

To monitor SINEC_Cf mobilization, we modified the Alu neo ${ }^{\text {tet }}$ vector, which contains an active human AluYa5 element equipped with a reporter cassette engineered to monitor the retrotransposition of RNA polymerase III (pol III) transcripts (75). Briefly, the Alu neo ${ }^{\text {tet }}$ vector consists of a 7SL RNA Pol III enhancer sequence upstream of AluYa5 that is equipped with a "backward" neo ${ }^{R}$ gene under the control of an SV40 promoter. The neo $o^{\mathrm{R}}$ gene is disrupted by a tetrahymena self-splicing group I intron that is in the same transcriptional orientation as the Alu element. This arrangement only allows the expression of the neo ${ }^{R}$ gene upon a successful round of retrotransposition in HeLa-HA cells, yielding G418-resistant foci (75). We replaced the AluYa5 sequence with the SINEC_Cf consensus sequence, obtained from Repbase (98). The resultant construct was used to assay SINEC_Cf mobilization, in trans, in the presence of either an active human LINE-1 or the newly cloned L1_Cf-104_5 expression plasmid that lacks the retrotransposition in-

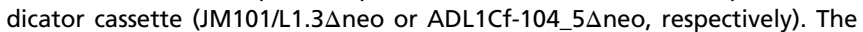
construction of the SINEC_Cf expression vector and the conditions used to assay for retrotransposition are detailed in SI Appendix, section 6.

Data Availability. Data have been deposited in National Center for Biotechnology Information GenBank database under accessions GCA 005444595.1, SWLD00000000.1, MK829534-MK829589, and MT811810; to the Sequence Read Archive under sample name SAMN04851098; and to the Gene Expression Omnibus database under accession GSE153608.

ACKNOWLEDGMENTS. This work was supported, in part, by NIH Grant R01GM103961 to J.M.K. and A.R.B., NIH Grant R01GM140135 to J.V.M. and J.M.K., NIH Academic Research Enhancement Award R15GM122028 to J.V.H., and NIH Training Fellowship T32HG00040 to A.L.P. DNA samples were provided by the Cornell Veterinary Biobank, a resource built with the support of NIH Grant R24GM082910, and the Cornell University College of Veterinary Medicine. Additional DNA samples were kindly provided by Brian Davis, Elaine Ostrander, and Linda Gates. We thank Dorina Twigg, Chai Fungtammasan, Brett Hannigan, Mark Mooney, Dylan Pollard, and DNAnexus for assistance with sequence data processing, and the University of Michigan Advanced Genomics Core for assistance with data production. We especially thank Linda Gates for her continued devotion to all Great Danes and assistance with this project. of coding genes and non-coding transcripts. PLoS One 9, e91172 (2014).

6. S. L. Ricketts, T. W. Marchant, Meeting report from the Companion Animal Genetic Health conference 2018 (CAGH 2018): A healthy companionship: The genetics of health in dogs. Canine Genet. Epidemiol. 5, 6 (2018).

7. L. Han, B. Su, W. H. Li, Z. Zhao, CpG island density and its correlations with genomic features in mammalian genomes. Genome Biol. 9, R79 (2008).

8. L. Han, Z. Zhao, Contrast features of $\mathrm{CpG}$ islands in the promoter and other regions in the dog genome. Genomics 94, 117-124 (2009). 
9. K. Paigen, P. Petkov, Mammalian recombination hot spots: Properties, control and evolution. Nat. Rev. Genet. 11, 221-233 (2010).

10. F. Baudat et al., PRDM9 is a major determinant of meiotic recombination hotspots in humans and mice. Science 327, 836-840 (2010).

11. S. Myers et al., Drive against hotspot motifs in primates implicates the PRDM9 gene in meiotic recombination. Science 327, 876-879 (2010).

12. E. D. Parvanov, P. M. Petkov, K. Paigen, Prdm9 controls activation of mammalian recombination hotspots. Science 327, 835 (2010).

13. L. Kauppi, A. J. Jeffreys, S. Keeney, Where the crossovers are: Recombination distributions in mammals. Nat. Rev. Genet. 5, 413-424 (2004).

14. L. Duret, N. Galtier, Biased gene conversion and the evolution of mammalian genomic landscapes. Annu. Rev. Genomics Hum. Genet. 10, 285-311 (2009).

15. J. Meunier, L. Duret, Recombination drives the evolution of GC-content in the human genome. Mol. Biol. Evol. 21, 984-990 (2004).

16. K. Paigen, P. M. Petkov, PRDM9 and its role in genetic recombination. Trends Genet 34, 291-300 (2018).

17. Z. Baker et al., Repeated losses of PRDM9-directed recombination despite the con servation of PRDM9 across vertebrates. eLife 6, e24133 (2017).

18. P. L. Oliver et al., Accelerated evolution of the Prdm9 speciation gene across diverse metazoan taxa. PLoS Genet. 5, e1000753 (2009).

19. E. Axelsson, M. T. Webster, A. Ratnakumar, C. P. Ponting, K. Lindblad-Toh; LUPA Consortium, Death of PRDM9 coincides with stabilization of the recombination landscape in the dog genome. Genome Res. 22, 51-63 (2012).

20. A. Auton et al., Genetic recombination is targeted towards gene promoter regions in dogs. PLoS Genet. 9, e1003984 (2013).

21. K. M. Credille et al., Transglutaminase 1-deficient recessive lamellar ichthyosis asso ciated with a LINE-1 insertion in Jack Russell terrier dogs. Br. J. Dermatol. 161, 265-272 (2009).

22. Z. T. Wolf et al., A LINE-1 insertion in DLX6 is responsible for cleft palate and mandibular abnormalities in a canine model of Pierre Robin sequence. PLoS Genet. 10, e1004257 (2014).

23. M. B. Brooks, W. Gu, J. L. Barnas, J. Ray, K. Ray, A Line 1 insertion in the Factor IX gene segregates with mild hemophilia B in dogs. Mamm. Genome 14, 788-795 (2003).

24. L. Lin et al., The sleep disorder canine narcolepsy is caused by a mutation in the hypocretin (orexin) receptor 2 gene. Cell 98, 365-376 (1999).

25. M. Pelé, L. Tiret, J. L. Kessler, S. Blot, J. J. Panthier, SINE exonic insertion in the PTPLA gene leads to multiple splicing defects and segregates with the autosomal recessive centronuclear myopathy in dogs. Hum. Mol. Genet. 14, 1417-1427 (2005).

26. L. A. Clark, J. M. Wahl, C. A. Rees, K. E. Murphy, Retrotransposon insertion in SILV is responsible for merle patterning of the domestic dog. Proc. Natl. Acad. Sci. U.S.A. 103 1376-1381 (2006)

27. N. B. Sutter et al., A single IGF1 allele is a major determinant of small size in dogs. Science 316, 112-115 (2007)

28. M. M. Gray, N. B. Sutter, E. A. Ostrander, R. K. Wayne, The IGF1 small dog haplotype is derived from Middle Eastern grey wolves. BMC Biol. 8, 16 (2010)

29. H. G. Parker et al., An expressed fgf4 retrogene is associated with breed-defining chondrodysplasia in domestic dogs. Science 325, 995-998 (2009).

30. L. M. Downs, C. S. Mellersh, An intronic SINE insertion in FAM161A that causes exonskipping is associated with progressive retinal atrophy in Tibetan Spaniels and Tibetan Terriers. PLoS One 9, e93990 (2014).

31. T. W. Marchant et al., Canine brachycephaly is associated with a retrotransposon mediated missplicing of SMOC2. Curr. Biol. 27, 1573-1584.e6 (2017).

32. E. A. Brown et al., FGF4 retrogene on CFA12 is responsible for chondrodystrophy and intervertebral disc disease in dogs. Proc. Natl. Acad. Sci. U.S.A. 114, 11476-11481 (2017).

33. W. Wang, E. F. Kirkness, Short interspersed elements (SINEs) are a major source of canine genomic diversity. Genome Res. 15, 1798-1808 (2005).

34. S. Boissinot, A. Entezam, L. Young, P. J. Munson, A. V. Furano, The insertional history of an active family of L1 retrotransposons in humans. Genome Res. 14, 1221-1231 (2004).

35. R. Everson et al., An intronic LINE-1 insertion in MERTK is strongly associated with retinopathy in Swedish Vallhund dogs. PLoS One 12, e0183021 (2017).

36. N. Katzir, E. Arman, D. Cohen, D. Givol, G. Rechavi, Common origin of transmissible venereal tumors (TVT) in dogs. Oncogene 1, 445-448 (1987)

37. C. Alkan, S. Sajjadian, E. E. Eichler, Limitations of next-generation genome sequence assembly. Nat. Methods 8, 61-65 (2011).

38. N. I. Weisenfeld, V. Kumar, P. Shah, D. M. Church, D. B. Jaffe, Direct determination of diploid genome sequences. Genome Res. 27, 757-767 (2017)

39. J. N. Burton et al., Chromosome-scale scaffolding of de novo genome assemblies based on chromatin interactions. Nat. Biotechnol. 31, 1119-1125 (2013).

40. N. Kaplan, J. Dekker, High-throughput genome scaffolding from in vivo DNA interaction frequency. Nat. Biotechnol. 31, 1143-1147 (2013).

41. C. S. Chin et al., Phased diploid genome assembly with single-molecule real-time sequencing. Nat. Methods 13, 1050-1054 (2016)

42. S. Koren et al., Canu: Scalable and accurate long-read assembly via adaptive $k$-mer weighting and repeat separation. Genome Res. 27, 722-736 (2017).

43. K. Sahlin, F. Vezzi, B. Nystedt, J. Lundeberg, L. Arvestad, BESST-Efficient scaffolding of large fragmented assemblies. BMC Bioinformatics 15, 281 (2014).

44. I. Numanagic et al., Fast characterization of segmental duplications in genome assemblies. Bioinformatics 34, i706-i714 (2018).

45. A. L. Pendleton et al., Comparison of village dog and wolf genomes highlights the role of the neural crest in dog domestication. BMC Biol. 16, 64 (2018).

46. M. R. Vollger et al., Long-read sequence and assembly of segmental duplications. Nat. Methods 16, 88-94 (2019).

47. W. J. Kent, BLAT-The BLAST-like alignment tool. Genome Res. 12, 656-664 (2002).
48. C. Trapnell et al., Differential gene and transcript expression analysis of RNA-seq experiments with TopHat and Cufflinks. Nat. Protoc. 7, 562-578 (2012)

49. C. Trapnell et al., Transcript assembly and quantification by RNA-Seq reveals unannotated transcripts and isoform switching during cell differentiation. Nat. Biotechnol. 28, 511-515 (2010)

50. M. G. Grabherr et al., Full-length transcriptome assembly from RNA-Seq data without a reference genome. Nat. Biotechnol. 29, 644-652 (2011).

51. V. Wucher et al., FEELnc: A tool for long non-coding RNA annotation and its application to the dog transcriptome. Nucleic Acids Res. 45, e57 (2017).

52. B. J. Raney et al., Track data hubs enable visualization of user-defined genome-wide annotations on the UCSC Genome Browser. Bioinformatics 30, 1003-1005 (2014).

53. L. A. Holden et al., Assembly and analysis of unmapped genome sequence reads reveal novel sequence and variation in dogs. Sci. Rep. 8, 10862 (2018).

54. V. P. Belancio, M. Whelton, P. Deininger, Requirements for polyadenylation at the 3 end of LINE-1 elements. Gene 390, 98-107 (2007).

55. M. A. Batzer et al., Structure and variability of recently inserted Alu family members. Nucleic Acids Res. 18, 6793-6798 (1990)

56. Q. Feng, J. V. Moran, H. H. Kazazian Jr., J. D. Boeke, Human L1 retrotransposon en codes a conserved endonuclease required for retrotransposition. Cell 87, 905-916 (1996)

57. G. J. Cost, J. D. Boeke, Targeting of human retrotransposon integration is directed by the specificity of the L1 endonuclease for regions of unusual DNA structure. Biochemistry 37, 18081-18093 (1998).

58. J. Jurka, Sequence patterns indicate an enzymatic involvement in integration of mammalian retroposons. Proc. Natl. Acad. Sci. U.S.A. 94, 1872-1877 (1997).

59. A. M. Roy et al., Potential gene conversion and source genes for recently integrated Alu elements. Genome Res. 10, 1485-1495 (2000).

60. M. A. Batzer, P. L. Deininger, Alu repeats and human genomic diversity. Nat. Rev. Genet. 3, 370-379 (2002).

61. D. H. Kass, M. A. Batzer, P. L. Deininger, Gene conversion as a secondary mechanism of short interspersed element (SINE) evolution. Mol. Cell. Biol. 15, 19-25 (1995).

62. A. M. Roy-Engel et al., Non-traditional Alu evolution and primate genomic diversity. J. Mol. Biol. 316, 1033-1040 (2002)

63. G.-D. Wang et al., Structural variation during dog domestication: Insights from gray wolf and dhole genomes. Natl. Sci. Rev. 6, 110-122 (2018).

64. C. Zhao, H. Zhang, G. Liu, X. Yang, J. Zhang, The complete mitochondrial genome of the Tibetan fox (Vulpes ferrilata) and implications for the phylogeny of Canidae. C. $R$. Biol. 339, 68-77 (2016).

65. H. Zhang, L. Chen, The complete mitochondrial genome of dhole Cuon alpinus: Phylogenetic analysis and dating evolutionary divergence within Canidae. Mol. Biol. Rep. 38, 1651-1660 (2011).

66. J. V. Moran, R. J. DeBerardinis, H. H. Kazazian Jr., Exon shuffling by L1 retrotransposition. Science 283, 1530-1534 (1999).

67. J. L. Goodier, E. M. Ostertag, H. H. Kazazian Jr., Transduction of 3'-flanking sequences is common in L1 retrotransposition. Hum. Mol. Genet. 9, 653-657 (2000).

68. O. K. Pickeral, W. Makałowski, M. S. Boguski, J. D. Boeke, Frequent human genomic DNA transduction driven by LINE-1 retrotransposition. Genome Res. 10, 411-415 (2000)

69. J. V. Moran, Human L1 retrotransposition: Insights and peculiarities learned from a cultured cell retrotransposition assay. Genetica 107, 39-51 (1999).

70. S. T. Szak, O. K. Pickeral, D. Landsman, J. D. Boeke, Identifying related L1 retrotransposons by analyzing 3' transduced sequences. Genome Biol. 4, R30 (2003).

71. C. M. Macfarlane et al., Transduction-specific ATLAS reveals a cohort of highly active L1 retrotransposons in human populations. Hum. Mutat. 34, 974-985 (2013).

72. H. C. Kopera et al., LINE-1 cultured cell retrotransposition assay. Methods Mol. Biol. 1400, 139-156 (2016)

73. J. V. Moran et al., High frequency retrotransposition in cultured mammalian cells. Cell 87, 917-927 (1996).

74. A. J. Doucet, J. E. Wilusz, T. Miyoshi, Y. Liu, J. V. Moran, A $3^{\prime}$ poly(A) tract is required for LINE-1 retrotransposition. Mol. Cell 60, 728-741 (2015).

75. M. Dewannieux, C. Esnault, T. Heidmann, LINE-mediated retrotransposition of marked Alu sequences. Nat. Genet. 35, 41-48 (2003).

76. M. O. Pollard, D. Gurdasani, A. J. Mentzer, T. Porter, M. S. Sandhu, Long reads: Their purpose and place. Hum. Mol. Genet. 27, R234-R241 (2018)

77. M. A. Field et al., Canfam_GSD: De novo chromosome-length genome assembly of the German Shepherd Dog (Canis lupus familiaris) using a combination of long reads, optical mapping, and Hi-C. Gigascience 9, giaa027 (2020).

78. C. Wang et al., A novel canine reference genome resolves genomic architecture and uncovers transcript complexity. Commun. Biol. 4 (2021).

79. J. Kieleczawa, Fundamentals of sequencing of difficult templates-An overview. J. Biomol. Tech. 17, 207-217 (2006)

80. G. Marais, Biased gene conversion: Implications for genome and sex evolution. Trends Genet. 19, 330-338 (2003)

81. M. J. P. Chaisson et al., Multi-platform discovery of haplotype-resolved structural variation in human genomes. Nat. Commun. 10, 1784 (2019).

82. H. L. Norton, E. E. Quillen, A. W. Bigham, L. N. Pearson, H. Dunsworth, Human race are not like dog breeds: Refuting a racist analogy. Evolution (N. Y.) 12, 17 (2019).

83. A. Auton et al.; 1000 Genomes Project Consortium, A global reference for human genetic variation. Nature 526, 68-74 (2015)

84. L. M. Payer et al., Structural variants caused by Alu insertions are associated with risks for many human diseases. Proc. Natl. Acad. Sci. U.S.A. 114, E3984-E3992 (2017)

85. W. Zhou et al., Identification and characterization of occult human-specific LINE-1 insertions using long-read sequencing technology. Nucleic Acids Res. 48, 1146-1163 (2020) 
86. D. Taliun et al., Sequencing of 53,831 diverse genomes from the NHLBI TOPMed Program. Nature 590, 290-299 (2021)

87. P. H. Sudmant et al.; 1000 Genomes Project Consortium, An integrated map of structural variation in 2,504 human genomes. Nature 526, 75-81 (2015).

88. K. H. Burns, Transposable elements in cancer. Nat. Rev. Cancer 17, 415-424 (2017).

89. S. Song, E. Sliwerska, S. Emery, J. M. Kidd, Modeling human population separation history using physically phased genomes. Genetics 205, 385-395 (2017).

90. D. Brown, B. Morgenstern, Algorithms in Bioinformatics: 14th International Work shop, WABI 2014, Wroclaw, Poland, September 8-10, 2014. Proceedings (Lecture Notes in Bioinformatics, Springer, New York, ed. 1, 2014), vol. 8701.

91. B. J. Haas et al., Improving the Arabidopsis genome annotation using maxima transcript alignment assemblies. Nucleic Acids Res. 31, 5654-5666 (2003).

92. B. J. Haas et al., De novo transcript sequence reconstruction from RNA-seq using the Trinity platform for reference generation and analysis. Nat. Protoc. 8, 1494-1512 (2013).
93. S. Götz et al., High-throughput functional annotation and data mining with the Blast2GO suite. Nucleic Acids Res. 36, 3420-3435 (2008).

94. N. L. Bray, H. Pimentel, P. Melsted, L. Pachter, Near-optimal probabilistic RNA-seq quantification. Nat. Biotechnol. 34, 525-527 (2016).

95. H. Li, Minimap2: Pairwise alignment for nucleotide sequences. Bioinformatics 34, 3094-3100 (2018).

96. A. Abyzov, M. Gerstein, AGE: Defining breakpoints of genomic structural variants at single-nucleotide resolution, through optimal alignments with gap excision. Bioinformatics 27, 595-603 (2011)

97. C. S. Chin et al., Nonhybrid, finished microbial genome assemblies from long-read SMRT sequencing data. Nat. Methods 10, 563-569 (2013).

98. W. Bao, K. K. Kojima, O. Kohany, Repbase Update, a database of repetitive elements in eukaryotic genomes. Mob. DNA 6, 11 (2015)

99. W. Wei et al., Human L1 retrotransposition: Cis preference versus trans complementation. Mol. Cell. Biol. 21, 1429-1439 (2001). 